\title{
Power Spectral Density Analysis of Speech Signal using Window Techniques
}

\author{
Jagriti Saini \\ ME Scholar, Department of Electronics and \\ Communication \\ National Institute of Technical Teachers Training \\ Chandigarh, India
}

\author{
Rajesh Mehra \\ Associate Professor, Department of Electronics \\ and Communication \\ National Institute of Technical Teachers Training \\ Chandigarh, India
}

\begin{abstract}
In this paper a comparative analysis of speech signal is performed using different window techniques. As each communication system consists of three major parts that are transmitter, receiver and channel. The level of power transmitted from transmitter end decides its ability to travel up to longer distance with minimum distortion. For analysis, first of all a signal out of audio frequency range is selected and then a small portion of this signal is extracted using framing technique. The resulting signal frame is passed through Hamming, Hanning and Blackman window and their respective power spectral densities are calculated. To analyze power content of signal Fast Fourier Transform is used. It can be obtained from the simulated results that Blackman window contains almost double power as compared to Hamming and Hanning window.
\end{abstract}

\section{General Terms}

Speech signal analysis, window techniques.

\section{Keywords}

Speech analysis; Power Spectral Density; Fast Fourier Transform; FIR; Hamming Window; Hanning Window; Blackman Window.

\section{INTRODUCTION}

Audio or speech signal is usually present in analog form but with tremendous growth in digital systems now most of applications are based on human computer interactions [1]. Human voice is in analog form but real time applications demand digital version of this data because it offers easy analysis, demands less storage space and can be transmitted up to longer distances with more security using various encryption-decryption [2] techniques. For analyzing performance of any audio signal over digital systems, the first most requirements is to convert analog information into some frames that can be passed through window function in order to judge its various parameters. In digital signal processing there are various windows that have different advantages as well as disadvantages over signal analysis; for making decision about which window is more suitable for certain audio application, it is good to perform comparative analysis. Major requirement for audio processing is to convert analog signal into digital signal with minimum or negligible losses at transmitter end so that actual information can be easily recovered at receiving end. For maximum extraction of signal at receiver it is important to ensure that transmitted signal contains enough power so that it can travel up to longer distanced through noisy channels and can deliver accurate information at receiver. Various Digital signal processing tools are used to maintain this power level of transmitted signal; the window techniques are most powerful solution to keep control over various parameters of signal so that its power level can be retained up to desired value.

This paper presents comparative analysis of Hamming, Hanning and Blackman window [2, 3] for audio signal processing. To judge their performance, some Matlab based simulations are performed for calculation of power spectral density of speech signal using different windows and with the help of Fast Fourier Transform, total power contained by each signal is calculated. In some applications, it is possible to compromise for power level of transmitted signals as in case of short distance communication but when it is desired to transmit data over longer distance, power becomes major requirement and it must be retained in all conditions. Application of Hamming, Hanning and Blackman window over framed version of speech signal resulted in different power levels. Hamming window provided least power whereas power level of signal that was processed through Blackman window was twice as that of Hamming. So for long distance communication it is more appropriate to use Blackman window so that signal with more power can be transmitted towards destination.

\section{WINDOW TECHNIQUES}

Window Techniques $[2,3]$ represents a function that is well known as Window Function or Tapering Function. This function states that application of Window over certain definite interval results in some finite and non-zero values inside that particular interval whereas it contains zero values outside that interval. There are so many window techniques that are useful in designing of FIR $[4,5,6]$ and IIR Filters. Most commonly used Windows for FIR Filter Designing are Hamming, Hanning and Blackman whereas for analysis of IIR Filters, it is preferred to use Butterworth and Chebyshev designs. For analysis of finite duration speech signal, following FIR Filter windows are used as:

\subsection{Hamming Window}

The Standard equation [3,5,6] for Hamming Window is defined as:

$\mathrm{w}(\mathrm{n})=\alpha-\beta \cos (2 \pi n / N-1))$ for $[-(N-1) / 2] \leq$ $n \leq[(N-1) / 2]$

With $\alpha=0.54$ and $\beta=1-\alpha=0.46$.

\subsection{Hanning Window}

The window function for causal Hanning Window [3, 5, 6] can be represented as:

WHann $(\mathrm{n})=\left\{\begin{array}{c}0.5-0.5 \cos \left(\frac{2 \pi n}{N-1}\right), 0 \leq n \leq N-1 \\ 0, \text { otherwise }\end{array}\right.$

Whereas non-causal Hanning Window function is given by: 


$$
\begin{aligned}
& \mathrm{W}_{\text {Hann }}(\mathrm{n})=\left\{0.5+0.5 \cos \left(\frac{2 \pi n}{N-1}\right), 0 \leq|n| \leq(N-1) / 2\right. \\
& 0 \text {, otherwise }
\end{aligned}
$$

\subsection{Blackman Window}

Blackman Window sequence $[3,5,6]$ is given as:

WBlack $(\mathrm{n})=\mathrm{a} 0+\mathrm{a} 1+\mathrm{a} 2 \cos (4 \pi \mathrm{n} /(\mathrm{N}-1))$ for $-(\mathrm{N}-1) / 2 \leq \mathrm{n} \leq(\mathrm{N}-$ $1) / 2$

Where a0, a1, a2 are constants:

$\mathrm{a} 0=(1-\alpha) / 2 ; \mathrm{a} 1=1 / 2 ; \mathrm{a} 2=\alpha / 2 ;$

All these windows play important role in Digital Signal Processing and help to analyze various parameters of signal.

\section{MATLAB BASED SIMULATIONS 3.1 Framing of Audio Signal}

To extract features of audio signal, frame blocking technique is implemented where continuous time audio signal is framed into $\mathrm{N}$ different samples. The adjacent frames in this sampled signal are separated by $\mathrm{M}$ samples where $\mathrm{M}<\mathrm{N}$ so that frame overlapping can be avoided. But for calculation of power level that any signal can retain after application of different windows it is enough to extract one single frame of $\mathrm{N}$ samples out of a continuous time audio signal and process it further using different windows. Fig.1.1 and Fig 1.2 represents audio signal and its framed version with $\mathrm{N}=20$, respectively.

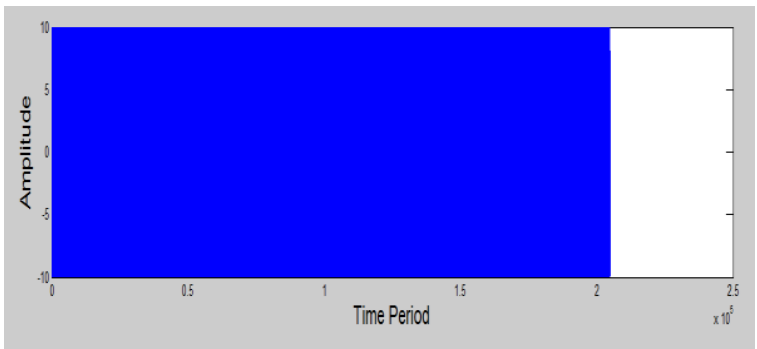

Fig 1.1: Input speech signal

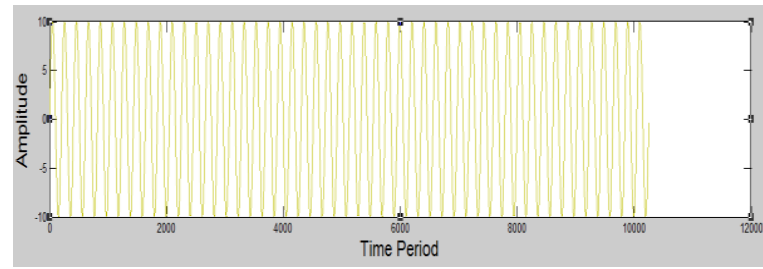

Fig. 1.2: Framed Signal

\subsection{Windowing}

The second step in signal analysis process is to window this framed signal in order to minimize discontinuities as well as to calculate width of central lobe that will contain most of signal power. As shown by fig.2, the width of central lobe after Hamming window $[2,3,5,6]$ is much wider whereas in case of Hanning window it is reduced by a very small amount. From simulated output it is observe that performance of Blackman window contributes into much steeper curve as compared to other two windows.

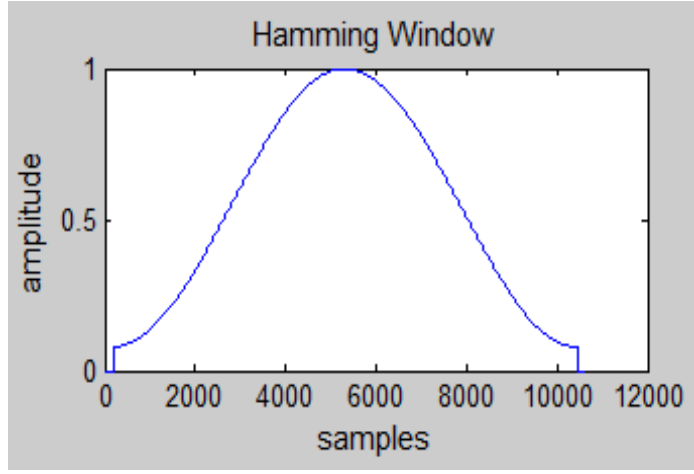

Fig. 2.1: Hamming Window Output.

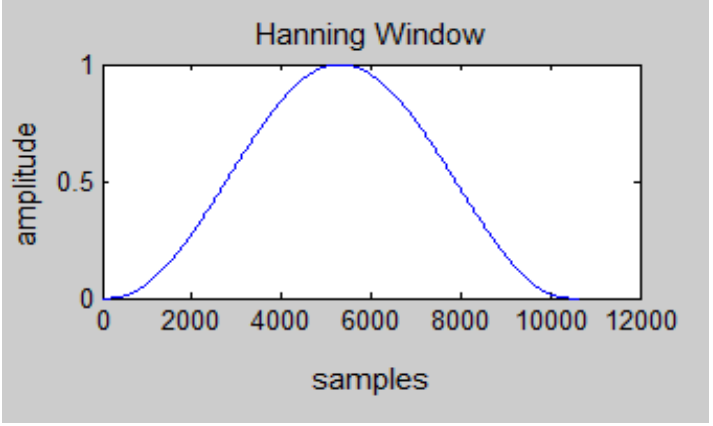

Fig. 2.2: Hanning Window Output.

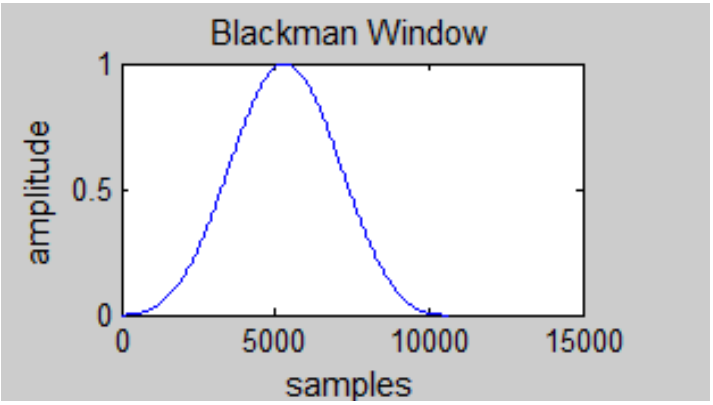

Fig2.3: Blackman Window Output.

\subsection{Power Spectral Density}

Power spectral density function helps to calculate the total power contained in each spectral component of certain signal. For example, consider a sine wave of some fixed frequency then PSD plot will incorporate only one spectral component that is present at that selected frequency. In simple words, power spectrum of any time domain signal $\mathrm{x}(\mathrm{t})$, helps to determine the distribution of variance of data $\mathrm{x}(\mathrm{t})$ over frequency domain in form of spectral components into which the actual signal can be decomposed. In this step, calculation of power spectral densities is done on signal obtained after application of different windows. Fig.3.1,3.2 and 3.3 Represents power spectral density plot for selected audio signal, its Hamming, Hanning and Blackman window result. 


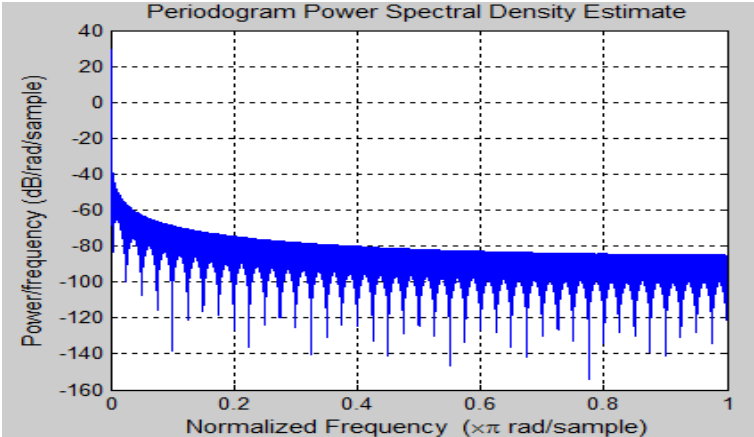

Fig.3.1: Power Spectral Density of Hamming Window.

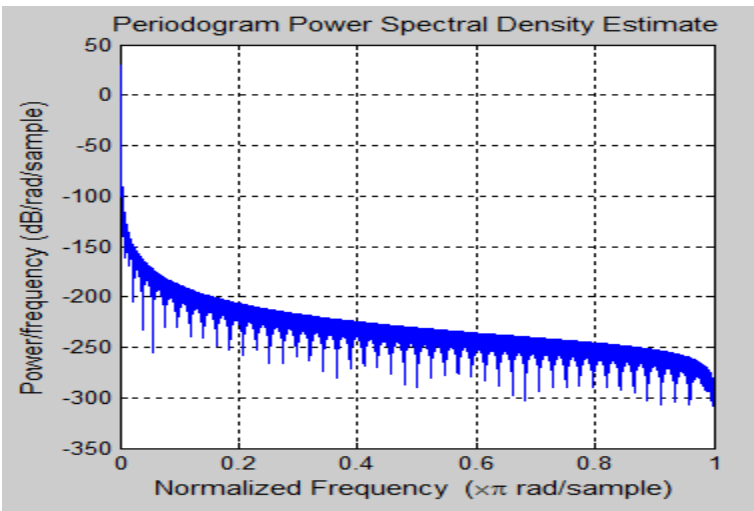

Fig.3.2: Power Spectral Density of Hanning Window.

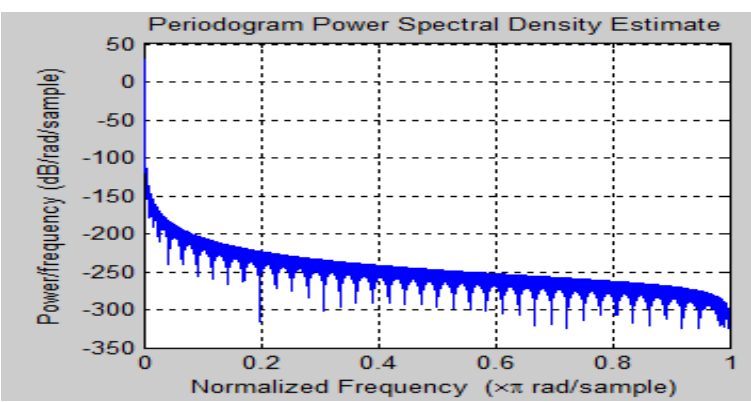

Fig.3.3: Power Spectral Density of Blackman Window.

\subsection{Fast Fourier Transform}

The next step of this digital signal processing is fast Fourier transform that provides conversion of framed signal from time domain into $\mathrm{N}$ samples leading toward frequency domain. FFT $[7,8]$ is useful tool in digital signal analysis because it helps to reduce computational complexity; the signal recovered after Fast Fourier transform is often called as periodogram which can be further utilized in calculation of power contained in signal under processing. Fig. 4 represents FFT results on signal recovered from different windows.

\subsection{Power Calculation}

Power calculation help to determine total power contained in signal under processing when it is passed through different windows. This power estimation helps to make analysis about which window is more suitable for audio applications depending upon distance between transmitter and receiver as well as conditions of channel. Signal containing more power can travel longer distances while suffering minimum losses. Fig.5 represents comparison of power contained by a frame of audio signal that is processed via different windows. It shows that power level of signal that was passed through Blackman window is more as compared to other two windows.

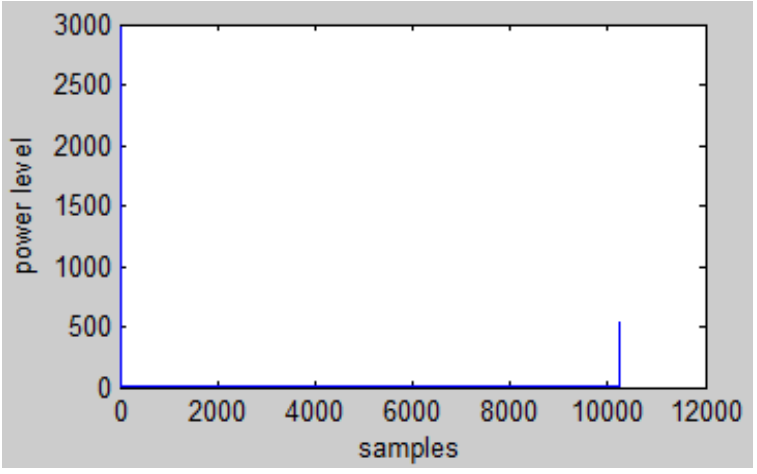

Fig. 4.1: Power Level of Hamming Window

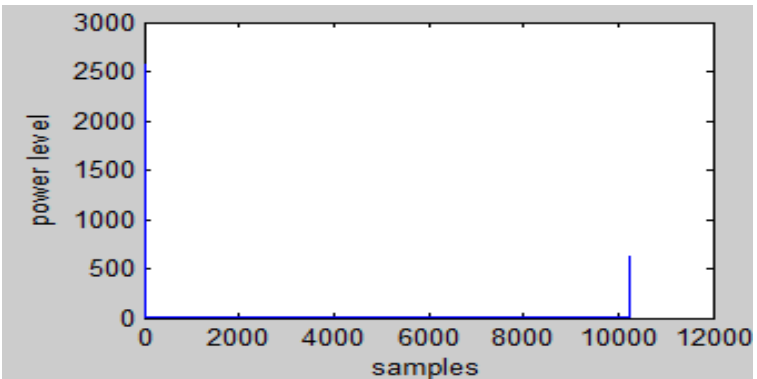

Fig. 4.2: Power level of Hanning Window

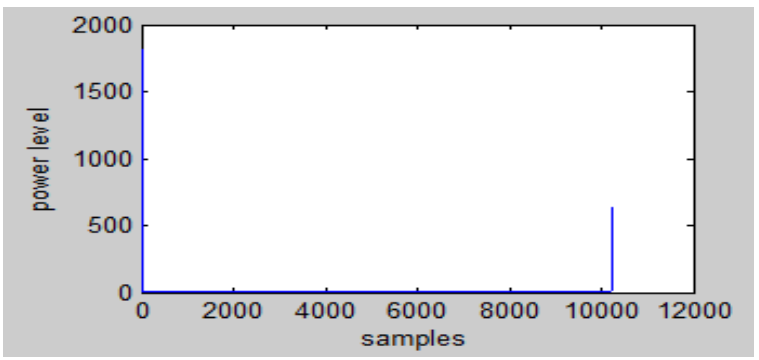

Fig. 4.3: Power level of Blackman Window

\section{RESULT ANALYSIS}

Matlab based simulation provides qualitative analysis for performance of different windows $[3,9,10,11]$ on speech signal. Ultimate goal is to make decision about suitable windows for different audio applications so that lossless communication can be achieved. These results help to analyze various factors of Hamming, Hanning and Blackman Windows.

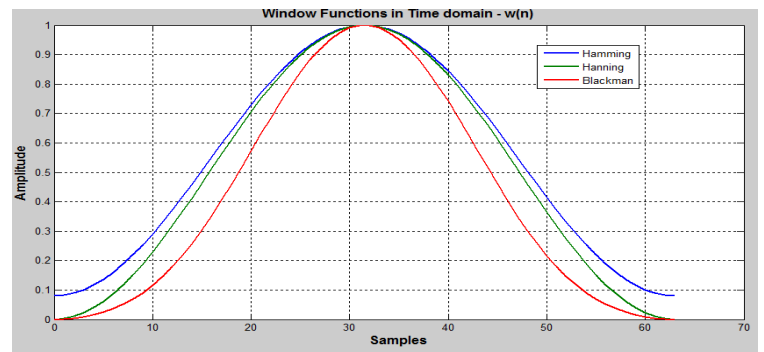

Fig.5: Comparison of different window outputs 


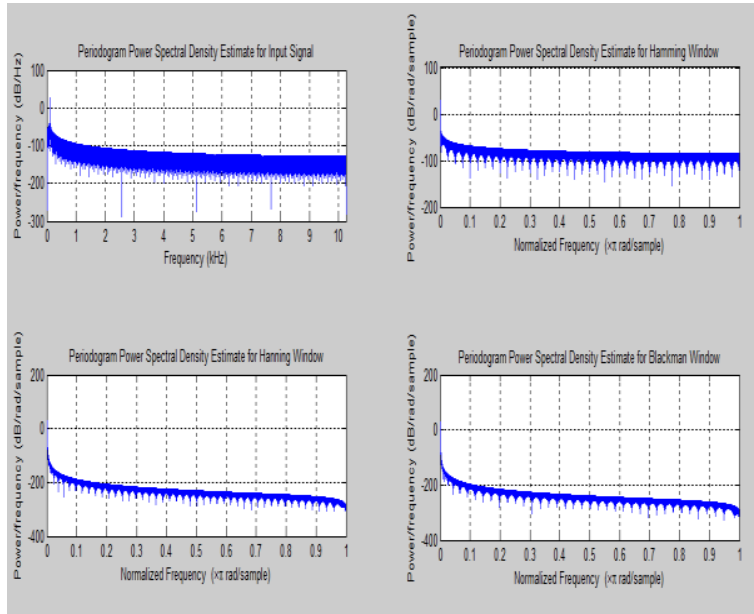

Fig.6: Comparison of power spectral densities for different windows.

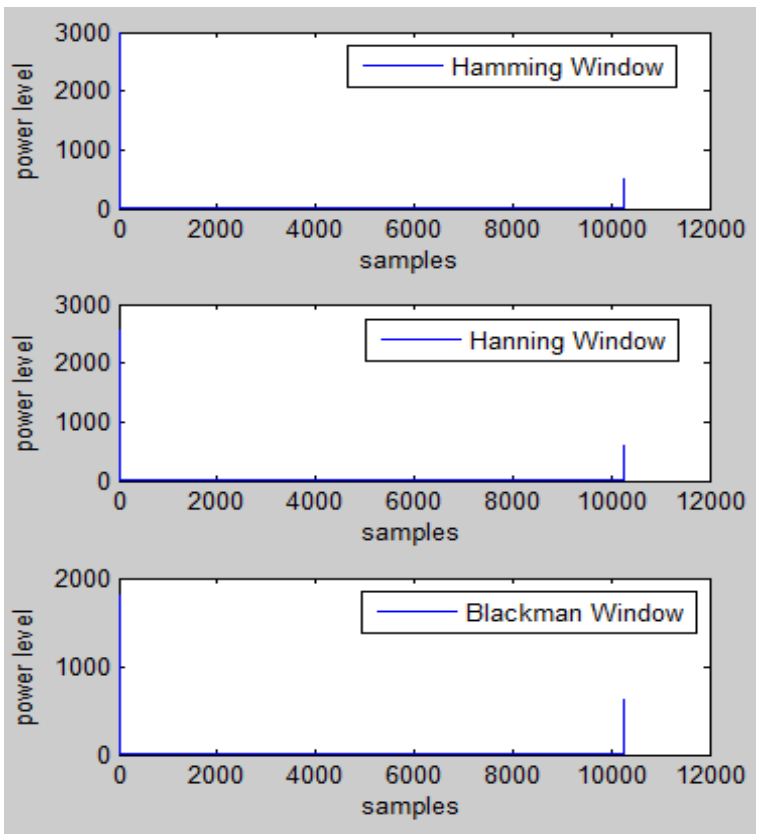

Fig.7: Comparison of Power Level for different windows.

\section{CONCLUSION}

This paper presents comparative analysis over performance of different windows [11] using audio signal as an input, it is observed that Blackman Window is more suitable for long distance communication because it delivers more power as compared to other two windows. The main lobe of Blackman window is steeper and its power spectral density is low as compared to hamming and Hanning window. With this analysis it can be said that Blackman Window is more suitable for long distance communication link based applications because this window will help signals to retain high power level during transmission so that they will get least affected by channel noise. This research work can be further extended towards creation of an advanced communication system by using some quality encoding, decoding, multiplexing and filtration techniques with Blackman window.

\section{ACKNOWLEDGMENT}

This research paper is outcome of continuous motivation and inspiration provided by Institute Director Dr. M. P. Poonia.

\section{REFERENCES}

[1] Mr. Maruti Saundade and Mr. Pandurang Kurle, Speech Signal Recognition using Digital Signal Processing, International Journal of Electronics Communication \& soft computing science and Engineering. Volume 2, Issue 6, pp: 31-34.

[2] Rajesh Mehra, Garima Saini, Sukhbir Singh. "FPGA based high speed $\mathrm{BCH}$ encoder for wireless communication applications". International Conference on Communication Systems and Network Technologies (CSNT), pp: 576-579. 3, June 2011

[3] Projoy Podder, Tanvir Zaman Khan, Haque Khan, M. Muktadir Rahman, "Comparative Performance Analysis of Hamming, Hanning and Blackman Window". International Journal of Computer Applications, Volume 96-No. 18, June 2014

[4] Kanu Priya, Rajesh Mehra. "Area Efficient Design of FIR Filter using Symmetric Structure". International Journal of Advanced Research in Computer and Communication Engineering. Volume 1, Issue 10, pp: 843-845.

[5] "Digital Signal Processing" third edition, John G Proakis, Dimitris G. Manolakis. Prentice Hall India. pp: 620-623.

[6] "Digital Signal Processing" second edition, S Salivahanan, C Gnanpriya. McGraw Hill. pp: 382-398, 435-464.

[7] [7] Pooja Kataria, Rajesh Mehra, "Comparative Analysis of FFT Algorithm for Different Window Techniques". International Journal of Science, Engineering and Technology Research (IJSETR), Volume 2, Issue 9, pp: 1691-1695. 2013

[8] Shaminder Kaur, Rajesh Mehra, "FPGA implementation of OFDM Transceiver using FFT Algorithm". International Journal of Engineering Science and Technology (IJEST), Volume 4 No. 04, pp: 1532-1537, April 2012.

[9] Mohd. Shariq Mahboob, Rajesh Mehra, "Design of Low Pass FIR Filter using Hamming, Blkackman- Harris and Taylor Window". International Journal of Advanced Research in Science and Engineering. Volume 3, Issue11, pp: 394-400. November 2014.

[10] Ritesh Pawar, Rajesh Mehra. "Design and Performance Analysis of FIR Filter for Audio Applications". International Journal of Science Research and Technology. pp: 122-126. August 2014.

[11] Saurabh Singh Rajput, Dr. S.S. Bhadauria. "Implementation of FIR Filter Using Efficient Window Function and its Application in Filtering a Speech Signal". International Journal of Electrical, Electronics and Mechanical Controls. Volume 1, Issue-1, November 2012. 